\title{
SpiderMAV: Perching and Stabilizing Micro Aerial Vehicles with Bio-inspired Tensile Anchoring Systems
}

\author{
K. Zhang, P. Chermprayong, T. M. Alhinai, R. Siddall, and M. Kovac
}

\begin{abstract}
Whilst Micro Aerial Vehicles (MAVs) possess a variety of promising capabilities, their high energy consumption severely limits applications where flight endurance is of high importance. Reducing energy usage is one of the main challenges in advancing aerial robot utility. To address this bottleneck in the development of unmanned aerial vehicle applications, this work proposes an bioinspired mechanical approach and develops an aerial robotic system for greater endurance enabled by low power station-keeping. The aerial robotic system consists of an multirotor MAV and anchoring modules capable of launching multiple tensile anchors to fixed structures in its operating envelope. The resulting tensile perch is capable of providing a mechanically stabilized mode for high accuracy operation in 3D workspace. We explore generalised geometric and static modelling of the stabilisation concept using screw theory. Following the analytical modelling of the integrated robotic system, the tensile anchoring modules employing high pressure gas actuation are designed, prototyped and then integrated to a quadrotor platform. The presented design is validated with experimental tests, demonstrating the stabilization capability even in a windy environment.
\end{abstract}

\section{INTRODUCTION}

Micro Aerial Vehicles (MAVs) have become an extensively developed part of the robotics landscape in the past few years [1]. However, the flight time of these aerial robots is severely limited to tens of minutes given current battery technology, and the maintenance of a stable position for precise operation is challenging, particularly in outdoor environment and partial failure conditions of the system. These main challenges [2] greatly reduce the efficacy of using MAVs to implement tasks requiring greater endurance and accuracy [3], such as close range aerial inspection and infrastructure reparation.

For the energy problem, perching has emerged as one of the most promising solutions, allowing aerial robots to operate in a low energy state between flights. A large number of concepts for perching MAVs have been presented, from both academic and commercial sources. This has included a variety of systems based on conventional pressure adhesives [4], spines and linkages for MAVs at smaller and larger scales respectively. Van der Waals dry adhesives [5], [6] employed in perching systems allow MAVs to stick to

\footnotetext{
*This work was supported by the Aerial ABM project grant from EPSRC UK under grant agreement EP/N018494/1.

K. Zhang, P. Chermprayong, T. M. Alhinai, R. Siddall and M. Kovac are with the Aerial Robotics Lab (ARL), Department of Aeronautics, Imperial College London, South Kensington Campus, London, SW7 2AZ United Kingdom ketao.zhangeimperial.ac.uk, pisak. chermprayong10@imperial.ac.uk,

r.siddall130imperial.ac.uk,

talib.al-hinail30imperial.ac.uk and

m. kovaceimperial.ac.uk
}

smooth vertical surfaces, while other systems employ spines to engage asperities on rough vertical wall surfaces [7]. Linkage based perching systems are capable of mimicking the agile perching behaviours of birds and allow both landing and take-off [8]. These systems all rely on a fixed attachment point, but the use multiple attach points with actuated tethers to allow a larger workspace within a movable operating envelope has been demonstrated in mobile camera systems such as SpyderCam.

All these systems shown that aerial robots using limited sensing and computation have benefited from perching to fixed structures, relying on mechanical intelligence to make up computational deficits at the small scale[2]. It has also demonstrated that perching can help to enhance capabilities of multimodal robots moving in cluttered environments [9], [10].

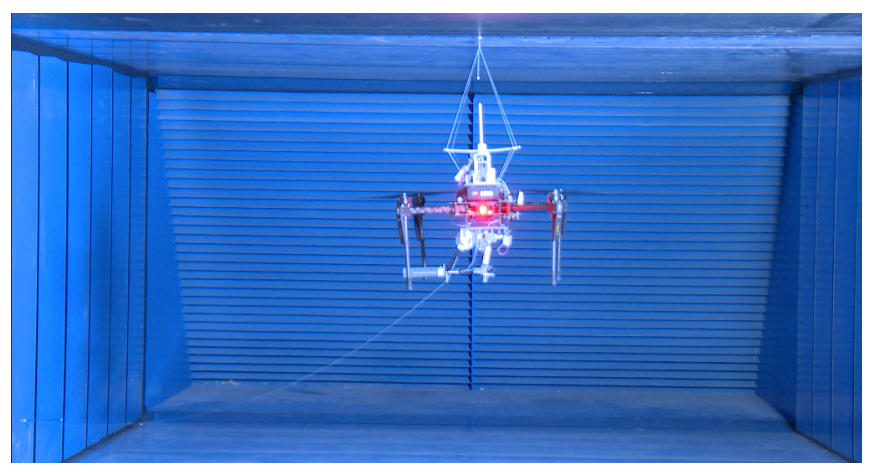

Fig. 1. The SpiderMAV: bio-inspired aerial robotic system capable of prolonged endurance and stabilized operations.

In parallel with approaches relying on mechanical intelligence, both sensing hardware and software control methods have also been continuously explored to enhance stabilization of MAVs in both manoeuvring and hovering. Complex estimation architectures with numerous embedded inertial sensors and cameras have proliferated for the purpose of stabilizing system performance in various flight modes [11], [12], and novel algorithms have been explored based on various platforms. These developments have included the stability margin evaluation method, [13], and the use of inertial optical flow in a nonlinear controller [14]. More recently, strategies for maintaining the controllability of quadrocopters under partial failure conditions have been investigated [15], [16], [17], leading to possible solutions for multicopter faulttolerant control design [18].

In nature, a spider is able to create large tensile structures between fixed attachments for predation and protection, and 
even passive flight ('ballooning' [19]). Taking inspiration [20] from this approach of using of tensile anchors for creating structures by the silk producing arachnids (Caerostris darwini), this paper proposes an innovative concept for developing multi-modal aerial robotic systems with perching and stabilizing capabilities, enabling solutions to critical challenges in MAVs operation such as endurance and accurate station keeping. In the following sections we will detail geometric and static design principles, explored in terms of screw theory and Grassmann line geometry. Based on this analysis, section III presents the design of modularized perching and stabilizing systems able to launch multiple tensile anchors onto distant structures, which are then integrated with a quadcopter platform and tested, showing the efficacy of the stabilization system (section IV). Finally, Section V discusses open questions and perspectives in this new trend of research.

\section{Geometric And Static Principles for Design AND ANALYSIS OF THE SPIDERMAV}

\section{A. The SpiderMAV}

The SpiderMAV proposed in this paper is an aerial robotic system inspired biologically by the web construction and locomotion capabilities of arachnids such as Darwin's Bark spider who spins strands of silk to build bridge lines up to 25 meters. While the spiders spray silk strands which drift on air current, the artificial SpiderMAV shoots threaded anchors from launchers allowing the anchor to reach fixed structures (ground bases) from distance and attach to targeted positions. The other end of each thread is wound around a spool mounted onto the MAV and can be actively coiled and uncoiled by the actuated spool. With threads in tension, the MAV in the absence of rotor thrusts is a platform suspended by a number of threads and subjects to pure forces including tensile forces provided by threads and the passive gravity. By rotating spools and adjusting length of each threads, the suspended MAV changes its position and orientation and manoeuvres in the 3D space. This type of thread/cable suspended systems are also referred to as wire/tendon-driven parallel mechanisms [21], [22], with characteristics such as large workspace, high load transmission and dynamic capacities useful in various applications.

\section{B. Geometric Modelling}

Without losing generality, a schematic diagram of the SpiderMAV in its station holding mode is illustrated in Fig. 2 where the system has $n$ rotors and $m$ threaded anchors attached to fixed structure. The point $A_{i}$ denotes the target position where an anchor attached while the point $B_{i}(i=$ $1,2, \ldots, m)$ denotes the other end of each thread connected to the multicopter frame. Here we assuming attaching points $B_{i}$ on the frame are located in a single plane $\Pi_{p}$ which is perpendicular to rotational axes of rotors $R_{j}(j=1,2, \ldots, n)$. Taking principle of kinematics, these points on both fixed structure and multicopter frame connecting the thread are equivalents of spherical joints.

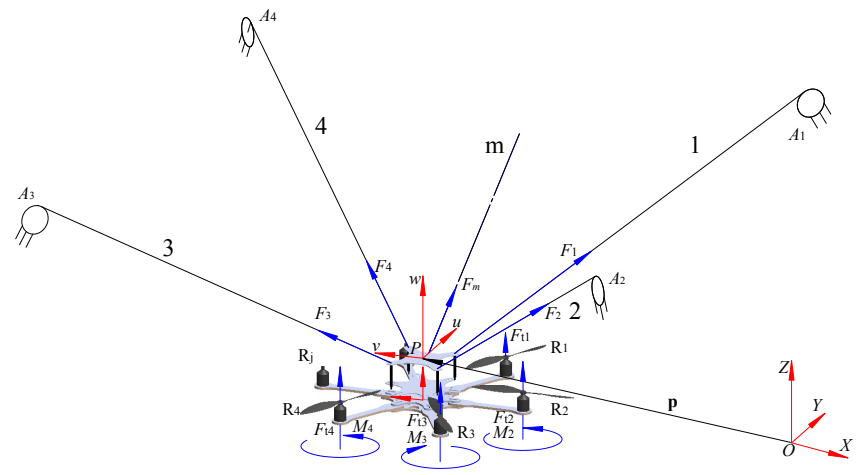

Fig. 2. Schematic representation of a station holding mode of the SpiderMAV.

An inertia reference frame $O-X Y Z$ is attached to the fixed structure. A moving reference frame $P-u v w$ is attached to the frame with origin $P$ located in the plane $\Pi_{p}$ and the $w$-axis perpendicular to the plane. For each thread, a local coordinate frame $B_{i}-x_{i} y_{i} z_{i}$ is set up with its origin attached at point $B_{i}$ by shifting reference frame $P-u v w$. Further, a local reference frame $Q-x^{\prime} y^{\prime} z^{\prime}$ is attached to the mass center $Q$ of the multicopter with $z$ '-axis aligning to $w$-axis by shifting reference frame $P-u v w$. For each rotor, a local coordinate frame $R_{j}-x_{j} y_{j} z_{j}$ is set up by shifting reference frame $Q-x^{\prime} y^{\prime} z^{\prime}$ and attaching the origin at point $R_{j}$.

In Fig.2, position vectors of string attaching points $B_{i}$ and rotor mounting points $R_{i}$ expressed in the moving reference frame $P-u v w$ are

$$
\left\{\begin{array}{l}
\mathbf{b}_{i}=\left[\begin{array}{lll}
x_{i} & y_{i} & 0
\end{array}\right]^{\mathrm{T}} \\
\mathbf{r}_{j}=\left[\begin{array}{lll}
x_{j} & y_{i} & h
\end{array}\right]^{\mathrm{T}}
\end{array}\right.
$$

where geometric parameters $x_{i}, y_{i}, x_{j}, y_{j}$ and $h$ are constants for a certain design of the SpiderMAV since the threads attaching points and the rotors mounting points are relative invariant on the multicopter frame.

\section{The Wrench Matrix of the System in a Stabilized Mode}

When the strings are in tension, $i$ th cable is considered as a line segment and the thread applies a pure force $F_{i}$ along the vector pointing from point $B_{i}$ to $A_{i}$. The pure force $F_{i}$ expressed in the local reference frame $B_{i}-x_{i} y_{i} z_{i}$ is a zero pitch screw vector which is defined as

$$
\mathbf{F}_{i}=t_{i}\left[\begin{array}{llllll}
a_{i} & b_{i} & c_{i} & 0 & 0 & 0
\end{array}\right]^{\mathrm{T}}
$$

where $t_{i}$ is the magnitude of the tensile force $F_{i}$ and $\left[a_{i}, b_{i}, c_{i}\right]^{\mathrm{T}}$ is the unit vector pointing from points $B_{i}$ to $A_{i}$. For thrust forces generated by rotors, each of them expressed in local frames $R_{j}-x_{j} y_{j} z_{j}$ are expressed as

$$
\mathbf{F}_{t j}=t_{j}\left[\begin{array}{llllll}
0 & 0 & 1 & 0 & 0 & 0
\end{array}\right]^{\mathrm{T}}
$$

in which vector $[0,0,1]^{\mathrm{T}}$ is the unit vector parallel to $z$ 'axis which represents the common direction of thrust forces by rotors while moments denoted by $\mathbf{M}_{j}$ are around rotors themselves and position free. In the moving coordinate frame, the moments are expressed as

$$
\mathbf{M}_{j}=t_{j}^{\prime}\left[\begin{array}{llllll}
0 & 0 & 0 & 0 & 0 & 1
\end{array}\right]^{\mathrm{T}}
$$


in which $t_{j}^{\prime}$ is the magnitude of the moment.

All the forces and moments applied to the integrated robotic system by threads and rotors expressed in the moving reference frame $P-u v w$ are derived by shifting,

$$
\mathbf{F}_{p k}=\mathbf{T}_{p k} \mathbf{F}_{k}
$$

where $k=i$ for cable tension exerting at points $B_{i}$ while $k=j$ for rotor thrust exerting at points $R_{j}$, and

$$
\mathbf{T}_{p k}=\left[\begin{array}{cc}
\mathbf{I} & 0 \\
\Delta_{p k} & \mathbf{I}
\end{array}\right]
$$

in which

$$
\Delta_{p i}=\left[\begin{array}{ccc}
0 & 0 & y_{i} \\
0 & 0 & -x_{i} \\
-y_{i} & x_{i} & 0
\end{array}\right]
$$

and

$$
\Delta_{p j}=\left[\begin{array}{ccc}
0 & -h & y_{j} \\
h & 0 & -x_{j} \\
-y_{j} & x_{j} & 0
\end{array}\right]
$$

Hence, the total active wrench $\mathbf{w}_{p}$ applied at point $P$ by the $m$ threads and the $n$ rotors is

$$
\mathbf{w}_{p}=\left[\begin{array}{lllllll}
\mathbf{F}_{p 1} & \mathbf{F}_{p 2} & \ldots & \mathbf{F}_{p k} & \mathbf{M}_{1} & \ldots & \mathbf{M}_{j}
\end{array}\right]
$$

Apart from forces and moments applied by threads and rotors, the system is also subject to a passive gravity force pointing in the inverse direction of $Z$-axis.

With the modelling of active wrench derived above, the disposition and number of threads in tension needed for mechanically stabilising a multicopter and avoiding singularity in the large workspace can be determined with consideration of the screw system in Eq.9. This wrench matrix is the basis to be used for determining the number and disposition of launchers in the design and system integration of a SpiderMAV in the following sections.

\section{Design of Modules for Perching AND STABILIZATION}

Following the geometric modelling and static analysis of the proposed robotic system for multi-modal locomotion in the section above, this section presents the design of modularized mechanisms for perching and stabilization with integrated anchor launchers. A common constraint for designing subsystems of MAVs is the limited payload, which means the perching and stabilizing modules in this work has to be lightweight. While the other technical challenges include efficient mechanisms allowing the system to actively launch anchors in various environments and system integration avoiding restrictions which limit the mobility of the MAV in certain workspace.

To allow the robot to actively fire tethered anchors (rather than drifting on air current in the manner bark spiders), the anchor launcher has to be able to release and retrieve strings, and to control the deployed length of uncoiled thread. In this work, magnets are used to attach the anchors to metallic structures. Given the relatively low mass of the quadcopter itself $(<2.5 \mathrm{~kg})$, the perching and stabilizing modules are not required to sustain heavy loads but to counter disturbances and ensure stability of the SpiderMAV in conditions such as a windy environment.

In summary the proposed perching and stabilizing modules need to have the following capabilities

- reconfiguring their structure and adjusting launching angles for targeting possible locations where magnetic anchors can attach;

- launching single/multiple threaded magnetic anchors for certain distances;

- controlling extension and maintaining tension of threads when magnetic anchors successfully attach to targets;

- retrieving threads in scenarios where the position and orientation of the system need to be reconfigured and the that magnetic anchor fails to attach to the target position.

Considering all constraints and functions required for perching and stabilizing modules, a lightweight pneumatic system capable of releasing compressed gas to launch threaded magnetic anchors is designed.
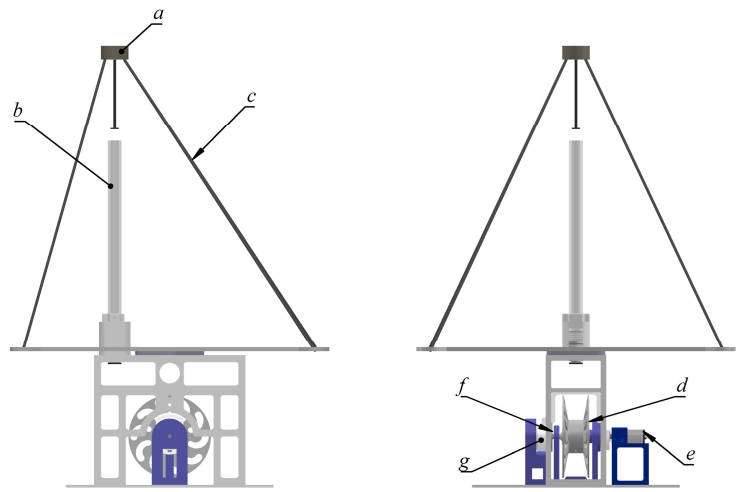

Fig. 3. The 3D model of perching module with a anchor launcher and string spooling system

\section{A. The Perching Module}

The perching module is to be mounted on the top of a quadcopter (DJI Matrice 100), allowing the integrated SpiderMAV to conduct Intelligence, Surveillance, and Reconnaissance (ISR) in a stationary holding mode with low energy consumption and prolonged endurance. The perching module consists of an anchor launcher and a spooling system shown in Fig.3.

The anchor $(a)$ is composed of a carbon fibre rod with $2 \mathrm{~mm}$ diameter, a ring magnet fixed at the top end of the rod and plastic ring disc at the bottom end. The permanent ring magnet with $20 \mathrm{~mm}$ outside diameter and $10 \mathrm{~mm}$ thickness is made of neodymium $(\mathrm{N} 42-\mathrm{NiCuNi})$ and capable of sustaining $9.4 \mathrm{~kg}$ pulling force axially. A polystyrene thread is tied to the magnetic ring. At the resting stage, the anchor is inserted into the aluminium barrel $(b)$ of which the bore diameter matches the outside diameter of the ring disc. The tolerance between the disc and the bore of barrel allows sliding motion with lower friction during launching stage. 
In the spooling system, a 3D printed spool $(d)$ is coupled with a micro-metal gearmotor $(e)$ and a ferrous shaft with a flat hat $(f)$, where the motor shaft and the ferrous shaft are coaxial. At the resting stage, the main section of the thread is wound on the spool while the remaining section coiled on the barrel. This allows the anchor to move freely without significant drag from the thread at the beginning of the launching stage. When the anchor successfully attaches to a target position, the motor then rotates in reverse direction to retrieve the string and change the longitude. In order to maintain the tension in the thread, a braking subsystem using magnetic force is adopted in this design. As shown in Fig. 4, a miniature electromagnet $(g)$ with a $20 \mathrm{~mm}$ outside diameter is mounted next to the flat hat of the ferrous shaft with a gap of less than $1 \mathrm{~mm}$ in the axial direction. When the electromagnet is powered, the motor is then turned off to avoid damage due to long period overloading. This electromagnet is capable of applying $2.5 \mathrm{~kg}$ pulling force when it is fully powered and providing forces to maintain the SpiderMAV at a desired stationary holding mode.

To launch the anchor, a solenoid valve triggers a quick exhaust valve to allow instant release of compressed gas stored in a 16 gram canister for accelerating the magnetic anchor while the plastic disc is pushing forward inside the barrel by pressured gas. The thread is carried by the anchor which then attaches to target position of fixed structures.

\section{B. A Reconfigurable Anchor Launcher for Stabilizing Mod- ule}

The 3D model of a reconfigurable anchor launcher employing a four-bar linkage for the stabilizing module is shown in Fig.4. It consists of three main subsystems including a anchor launcher, a spool actuation and braking system, and a planar crank-slider mechanism for adjusting the angle the launcher.

The anchor $(a)$ in Fig. 4 is composed of a carbon fibre rod with $2 \mathrm{~mm}$ diameter, a ring neodymium magnet (N42$\mathrm{NiCuNi}$ ) with $6 \mathrm{~mm}$ outside diameter and $2 \mathrm{~mm}$ diameter hole fixed at one end of the rod and a plastic disc at the other end of the rod. The ring magnet is capable of holding $1.3 \mathrm{Kg}$ pulling force. The total weight of the anchor is 20 grams. By varying the number or diameters of ring magnets, the weight and capability of the pulling force of the anchor can be changed for different requirements. The customized barrel is connected to the gas canister with a pneumatic elbow tube adapter $(c)$ capable of maximum operating pressure at 10 bar.

The reconfigurable anchor launcher uses same spool actuating and braking system for the perching module, of which the elements are denoted by $(d),(e),(f)$ and $(g)$.

The above two subsystems are integrated in a planar crank-slider mechanism where the slider is actuated with a customized linear screw drive system $(h)$. The crank $(i)$ is rotating around the coaxes of the motor and ferrous shaft at one end while the other end is jointed to a bracket mounted on the barrel $(b)$. Coupler $(j)$ is jointed to the bracket and linear screw drive system $(h)$. With this one degreeof-freedom planar mechanism, anchor launching angle is

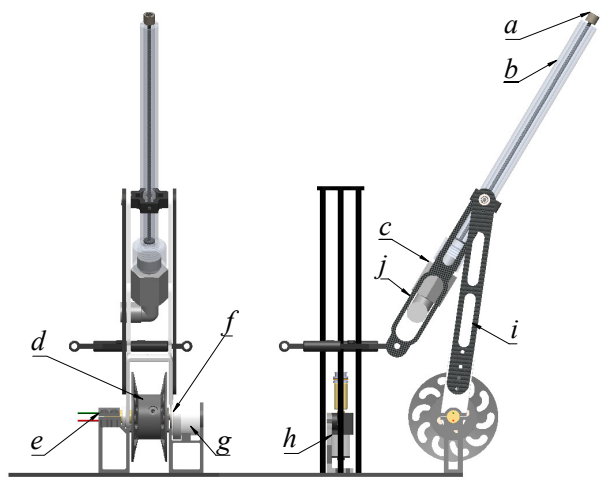

Fig. 4. Front and side views of 3D model of the four-bar linkage based reconfigurable anchor launcher

changeable and provide extra flexibility for the stabilizing module to aim at target position. The design parameters of the mechanism are customized to allow the barrel to change its direction with in a domain $[0,120]$ deg measured from axis of the linear screw drive system $(h)$.

\section{The Integrated Stabilizing Module}

Stabilizing modules with various number of anchor launchers can be configured following the principle in Section II, allowing all integrated planar mechanisms to be driven by one common linear screw drive system as shown in Fig.5.

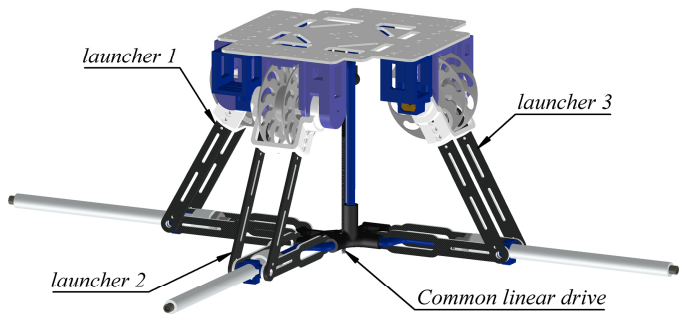

Fig. 5. An assembly of a stabilizing module with three anchor launcher units sharing a common linear drive

For a quadcopter in a hovering mode, the thrust forces which are all in parallel applied by rotors form a screw system of order 3 . Based on the Grassmann varieties, a SpiderMAV formed with a quadrotor needs at least three more independent forces from threads to achieve a fully constrained configuration for enhanced stabilization in the workspace.

Following this, two prototypes were built for validating the design of the SpiderMAV proposed in this paper. One prototype with pure stabilizing module composed of three anchor launchers and another prototype with one perching module on the top and one stabilizing module composed of two anchor launchers at the bottom of the quadcopter as shown in Fig. 6, and the video attachment.

\section{Electronics and Control}

The control system of the perching and stabilizing modules consists of 6 subsystems: power management, wireless com- 


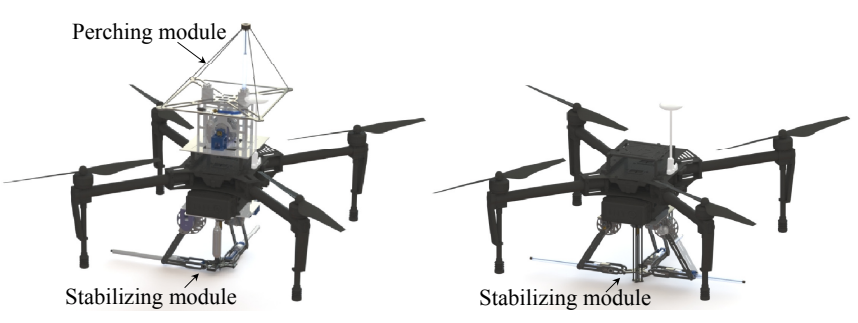

Fig. 6. The SpiderMAV: the 3D model of designs with both perching and stabilizing modules (the left-hand side) and only stabilizing module (the right-hand side)

munication, solenoid valve actuation system, linear drive, spooling motor control and electromagnetic braking system. For the processing unit, the Adafruit Feather M0 microcontroller is utilized to control all subsystems of the SpiderMAV.

UART over Bluetooth has been chosen to be a communication protocol to actuate devices in each subsystem. Each electromagnet of the braking system is actuated by a separate MOSFET, leading to braking process for individual electromagnet or all at once. Both linear drive actuation system and spooling motor control employ bi-directional motor drivers, allowing robust control of the actuators in both directions. In the current setting, the Adafruit Fether M0 micrcontroller is powered by a separate $1 \mathrm{~S}$ lithium-polymer battery and all other devices are powered by the battery of the Matrice 100. The electronics diagram for the control system is illustrated in Fig.7

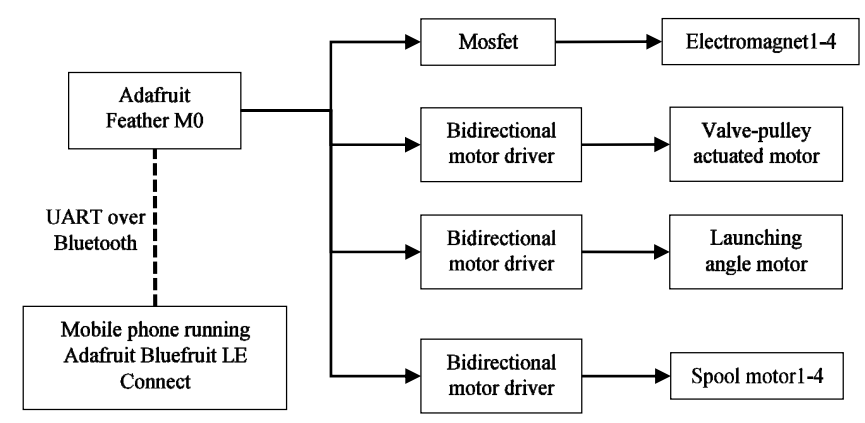

Fig. 7. The electronics diagram for the control system

\section{PRototypes ANd Experimental VALidATION OF ENHANCED STABILITY OF STATION HOLdING WiTH THREADED ANCHORS}

In this section, experiments for characterizing the threaded anchor launcher as well as enhanced stability of the selected quadcopter, DJI Matrice 100, with a stabilizing module are presented.

In order to obtain horizontal barrel exit speed of the anchor, tests were implemented with a launcher powered by compressed gas at various pressures and horizontal barrel axis. The projection of the anchor is then captured with a fast speed video camera (FASTEC-TS5-386). The barrel exit velocities for gas pressures at the range of $[4,6]$ bar were then tracked in software using stack images captured in experiments. The results of barrel exit velocities in Fig.8 guide settings of gas pressure for the anchoring system to allow the anchor to reach a target position which is 2.6 meter away from the barrel exit.

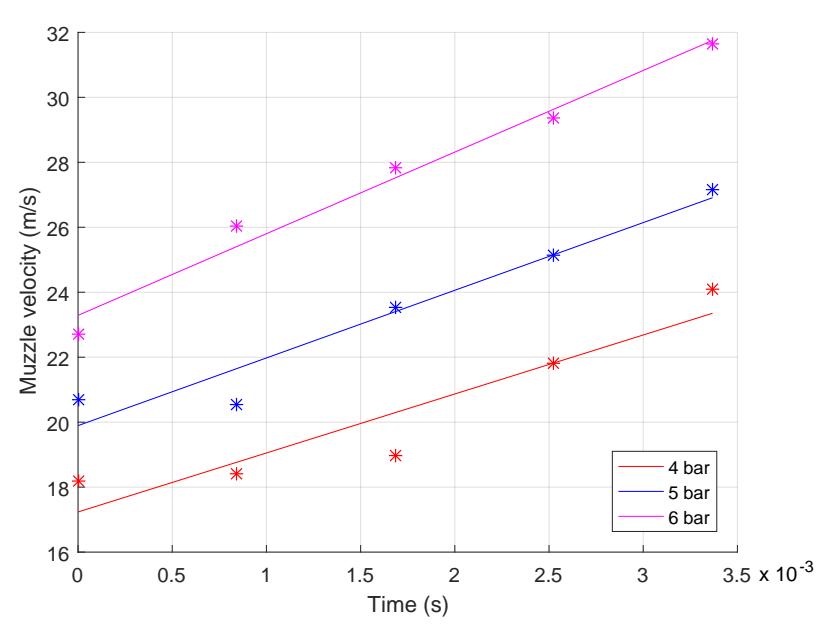

Fig. 8. Barrel exit speed of the anchor with various pressure applied to the launcher.

Tests were conducted to validate the performance of SpiderMAVs. The first prototype with both perching and stabilizing modules were tested in different environments with metallic surfaces at different corrosion states. As shown in the video attachment, the magnetic anchor launched by perching module was able to attach to the ceiling frame, thus allowing the robot to hold its position and orientation at high attitude and save onboard energy with rotors spinning in lower speed then switched off.

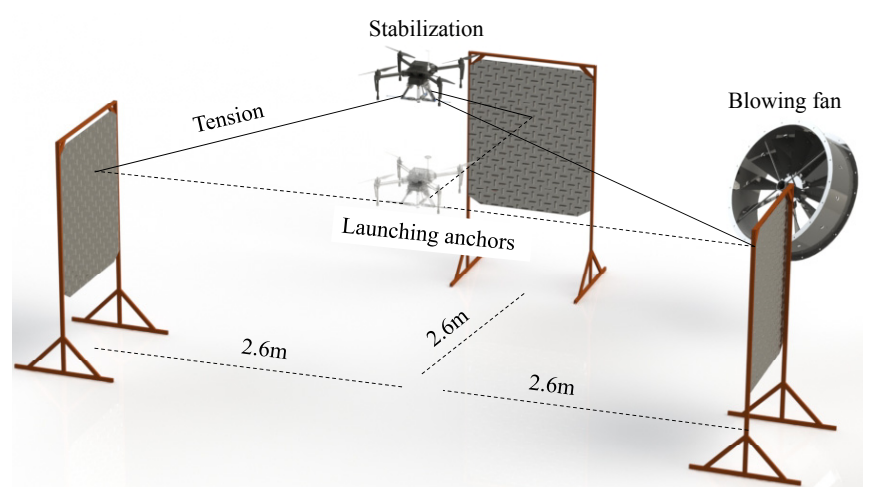

Fig. 9. Testing setup for validating enhanced stabilization of the SpiderMAV in windy condition.

To evaluate the performance for enhanced stabilization of the SpiderMAVs, the second prototype with only stabilizing module was tested in attitude-hold mode for hovering the MAV at the same position and orientation against one Drum Electric Fan that was blowing and generating 5500cfm air flow directly at the MAV as illustrated in Fig.9. The anchors were then launched and attached to vertical metallic plates in three directions. With all the anchors successfully attached to the plates, the spooling system coiled the threads back to get them in tension and hence initiated the stabilizing 
mechanism. Vicon motion capture system was used to log the position of the MAV to quantify and compare the drift in 3-dimensions space of the MAV with and without the stabilizing module.

While attitude-hold mode control of the DJI M100 gave maximum deviation of 136, 386 and $106 \mathrm{~mm}$ in $\mathrm{X}, \mathrm{Y}$ and $\mathrm{Z}$ direction respectively, test results showed that the stabilizing module improved stability of the MAV in all 3-dimensions with maximum deviation of 47,80 and $75 \mathrm{~mm}$ in $\mathrm{X}, \mathrm{Y}$ and $\mathrm{Z}$ respectively as indicated in Fig.10.

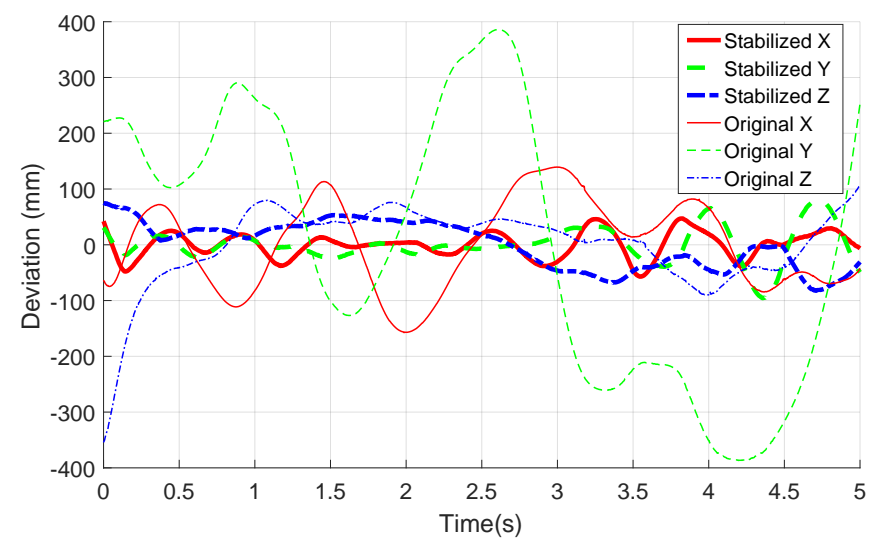

Fig. 10. Comparison of stability of the original DJI Matrice 100 and the SpiderMAV both in attitude-hold control mode.

\section{CONCLUSIONS}

The work presented in this paper tackles limited flight endurance and unexpected drift, which have been two of the main challenges for aerial robots by exploring biologically approaches in nature. Taking inspiration from the approach of using silk strands for creating large tensile structures by Darwin's bark spider, this paper proposed an innovative concept for developing multi-modal aerial robotic systems with perching and stabilizing capabilities, enabling solutions to critical challenges in MAVs operation such as endurance and accurate station keeping. With this bio-inspired concept, a tensile anchoring system was designed and employed in both perching and stabilising modules for low power stationkeeping and multi-modal operation. Using screw theory and Grassmann varieties, geometric and static principles of the aerial robotic system with both anchored tensile strings and thrusts generated by rotors were explored for modelling of the integrated system. The anchoring system powered by pressed gas has been characterized, allowing the anchor to reach target positions at certain distance with optimized pressure. The presented design concept for perching and stabilization were then validated with experimental tests of prototypes developed, demonstrating the operation of the integrated SpiderMAV in a enhanced stable mode comparing to pure control based performance.

The proposed robotic system also opens up a new trend in the field of robotics on investigating string driven systems with active moving platform. Analytical modelling of kine- matics, statics and dynamics during locomotion are essential open problems for such an interesting system.

\section{ACKNOWLEDGMENT}

The authors thank Mr Roland Hutchins for machining mechanical parts of the robot.

\section{REFERENCES}

[1] D. Floreano and R. J. Wood, "Science, technology and the future of small autonomous drones," Nature, vol. 521, no. 7553, pp. 460-466, 2015.

[2] M. Kovac, "Learning from nature how to land aerial robots," Science, vol. 352, no. 6288, pp. 895-896, 2016.

[3] G. Hunt, F. Mitzalis, T. Alhinai, P. A. Hooper, and M. Kovac, "3d printing with flying robots," in Robotics and Automation (ICRA), 2014 IEEE International Conference on. IEEE, 2014, pp. 4493-4499.

[4] M. Anderson, "The sticky-pad plane and other innovative concepts for perching uavs," in 47th AIAA Aerospace Sciences Meeting Including The New Horizons Forum and Aerospace Exposition, 2009, p. 40.

[5] A. Kalantari, K. Mahajan, D. Ruffatto, and M. Spenko, "Autonomous perching and take-off on vertical walls for a quadrotor micro air vehicle," in Robotics and Automation (ICRA), 2015 IEEE International Conference on. IEEE, 2015, pp. 4669-4674.

[6] E. W. Hawkes, H. Jiang, and M. R. Cutkosky, "Three-dimensional dynamic surface grasping with dry adhesion," The International Journal of Robotics Research, vol. 35, no. 8, pp. 943-958, 2016.

[7] A. Lussier Desbiens, A. T. Asbeck, and M. R. Cutkosky, "Landing, perching and taking off from vertical surfaces," The International Journal of Robotics Research, vol. 30, no. 3, pp. 355-370, 2011.

[8] P. Xie, O. Ma, and Z. Zhang, "A bio-inspired approach for uav landing and perching," in AIAA Guidance, Navigation, and Control (GNC) Conference, 2013, p. 5108.

[9] M. Kovač, J. Germann, C. Hürzeler, R. Y. Siegwart, and D. Floreano, "A perching mechanism for micro aerial vehicles," Journal of MicroNano Mechatronics, vol. 5, no. 3-4, pp. 77-91, 2009.

[10] M. Kovač, O. Fauria, J.-C. Zufferey, D. Floreano et al., "The locomotion capabilities of the epfl jumpglider: A hybrid jumping and gliding robot," in Robotics and Biomimetics (ROBIO), 2011 IEEE International Conference on. IEEE, 2011, pp. 2249-2250.

[11] P.-J. Bristeau, F. Callou, D. Vissiere, and N. Petit, "The navigation and control technology inside the ar. drone micro uav," IFAC Proceedings, vol. 44, no. 1, pp. 1477-1484, 2011.

[12] T. Krajník, V. Vonásek, D. Fišer, and J. Faigl, “Ar-drone as a platform for robotic research and education," in International Conference on Research and Education in Robotics. Springer, 2011, pp. 172-186.

[13] V. Mukhopadhyay and J. Newsom, "A multiloop system stability margin study using matrix singular values," Journal of Guidance, Control, and Dynamics, vol. 7, no. 5, pp. 582-587, 1984.

[14] B. Herisse, F.-X. Russotto, T. Hamel, and R. Mahony, "Hovering flight and vertical landing control of a vtol unmanned aerial vehicle using optical flow," in Intelligent Robots and Systems, 2008. IROS 2008. IEEE/RSJ International Conference on. IEEE, 2008, pp. 801-806.

[15] M. Ranjbaran and K. Khorasani, "Fault recovery of an under-actuated quadrotor aerial vehicle," in Decision and Control (CDC), 2010 49th IEEE Conference on. IEEE, 2010, pp. 4385-4392.

[16] H. A. Izadi, Y. Zhang, and B. W. Gordon, "Fault tolerant model predictive control of quad-rotor helicopters with actuator fault estimation," IFAC Proceedings Volumes, vol. 44, no. 1, pp. 6343-6348, 2011.

[17] A. Freddi, A. Lanzon, and S. Longhi, "A feedback linearization approach to fault tolerance in quadrotor vehicles," IFAC Proceedings Volumes, vol. 44, no. 1, pp. 5413-5418, 2011.

[18] M. W. Mueller and R. D'Andrea, "Stability and control of a quadrocopter despite the complete loss of one, two, or three propellers," in Robotics and Automation (ICRA), 2014 IEEE International Conference on. IEEE, 2014, pp. 45-52.

[19] J. Humphrey, "Fluid mechanic constraints on spider ballooning," Oecologia, vol. 73, no. 3, pp. 469-477, 1987.

[20] M. Kovač, "The bioinspiration design paradigm: A perspective for soft robotics," Soft Robotics, vol. 1, no. 1, pp. 28-37, 2014.

[21] A. Pott and T. Bruckmann, Cable-Driven Parallel Robots. Springer, 2013.

[22] C. GOSSELIN, "Cable-driven parallel mechanisms: state of the art and perspectives," Mechanical Engineering Reviews, vol. 1, no. 1, pp. DSM0004-DSM0004, 2014. 